\title{
Nuclear VEGFR-2 Expression of Hepatocytes Is Involved in Hepatocyte Proliferation and Liver Regeneration During Chronic Liver Injury
}

\author{
A-RANG LEE ${ }^{1}$, SU-MIN BAEK ${ }^{1}$, SEOUNG-WOO LEE $^{1}$, TAE-UN KIM ${ }^{1}$, \\ JEE EUN HAN ${ }^{2}$, SEULGI BAE ${ }^{2}$, SANG-JOON PARK ${ }^{2}$, TAE-HWAN KIM ${ }^{1}$, \\ KYU-SHIK JEONG ${ }^{1,3}$, SEONG-KYOON CHOI ${ }^{4}$ and JIN-KYU PARK ${ }^{1,3}$ \\ ${ }^{1}$ Department of Veterinary Pathology, College of Veterinary Medicine, \\ Kyungpook National University, Daegu, Republic of Korea; \\ ${ }^{2}$ College of Veterinary Medicine, Kyungpook National University, Daegu, Republic of Korea; \\ ${ }^{3}$ Stem Cell Therapeutic Research Institute, Kyungpook National University, Daegu, Republic of Korea; \\ ${ }^{4}$ Core Protein Resources Center, Daegu Gyeongbuk Institute of Science \\ and Technology (DGIST), Daegu, Republic of Korea
}

\begin{abstract}
Background/Aim: The pathological role of vascular endothelial growth factor receptor 2 (VEGFR-2) in chronic liver injury and liver regeneration is not fully understood. This study analysed the role of VEGFR-2 in liver fibrosis and its regeneration process. Materials and Methods: We administered $50 \mathrm{mg} / \mathrm{kg}$ to $300 \mathrm{mg} / \mathrm{kg}$ thioacetamide (TAA) to 9-week-old male mice intraperitoneally for 17 weeks. We measured the levels of VEGFR-2 protein and identified the location of cells that specifically express VEGFR-2. Results: VEGFR-2 is rarely expressed in normal hepatocytes. However, high VEGFR-2 expression in liver sinusoidal endothelial cells was noted in the TAA group. Conversely, the group that experienced regeneration from liver fibrosis showed significantly higher VEGFR-2 expression in the nucleus of hepatocytes compared to the other groups. Conclusion: VEGFR-2 plays a pivotal role in the nucleus of hepatocytes during liver regeneration and VEGFR-2 may be
\end{abstract}

This article is freely accessible online.

Correspondence to: Jin-Kyu Park, DVM, Ph.D., Department of Veterinary Pathology, College of Veterinary Medicine, Kyungpook National University, 80, Daehak-ro 9-gil, Buk-gu, Daegu 41566, Republic of Korea. Tel: +82 539505962, e-mail: jinkyu820@knu.ac.kr and Seong-Kyoon Choi, DVM, Ph.D., Core Protein Resources Center, Daegu Gyeongbuk Institute of Science and Technology (DGIST), 333, Techno jungang-daero, Hyeonpungeup, Dalseong-gun, Daegu 42988, Republic of Korea. Tel: +82 537852562, e-mail: cskbest@dgist.ac.kr

Key Words: VEGFR-2, cell proliferation, liver regeneration, chronic liver disease, intracellular location. closely related to cell division. Therefore, VEGFR-2 may be a new therapeutic target for liver regeneration.

Liver fibrosis is marked by the formation of an abnormally large amount of scar tissue in the liver (1). Liver fibrosis represents the final common route of chronic liver disease, which eventually leads to cirrhosis (2). Chronic liver disease and cirrhosis result in approximately 35,000 deaths yearly in the United States and cirrhosis is the ninth leading cause of death (3). Therefore, it is very important that liver fibrosis does not progress to cirrhosis, an irreversible stage.

Angiogenesis, which is the process of new blood vessel growth from existing vessels, is essential for the physiological functioning of tissues (4). Hepatic angiogenesis is closely associated with the progression of fibrosis in chronic liver diseases (5). According to a recent study, hepatic angiogenesis occurs in chronic liver disease, and is characterized by progressive fibrosis (6). Angiogenesis is a dynamic process regulated by the balance between pro-angiogenic and antiangiogenic factors. Vascular endothelial growth factor (VEGF) is one of the most important pro-angiogenic factors (4).

Hepatocytes play a key role in angiogenesis, and have a close anatomical relationship with endothelial cells that secrete VEGF (7). VEGF regulates blood and lymphatic vessel development and maintains homeostasis (8). VEGF is primarily produced by endothelial cells in response to hypoxia and by stimulation of growth factors such as transforming growth factor, interleukins or platelet derived growth factor (9, 10). The biological function of VEGF is mediated upon binding to type III receptor tyrosine kinase (RTK), VEGF receptor 1 (VEGFR-1, Flt-1), VEGFR-2 (KDR/Flk-1), and VEGFR-3 (Flt-4) $(11,12)$. VEGF stimulates angiogenesis by 
binding to VEGFR, which is also a type of RTK, on the cell surface of endothelial cells (13). Generally, steady state hepatocytes are not known to express VEGFR-2 in the normal liver $(14,15)$. However, the exact role of VEGFR-2 in the liver, particularly in relation to hepatocytes, is not fully understood.

VEGFR-2 is the primary responder to VEGF signals; thus, it regulates endothelial migration and proliferation (16). In general, VEGF is released by hepatocytes and endothelial cells during hypoxic conditions or by damaged macrophages, which bind to VEGFR on the cytoplasmic membrane of liver sinusoidal endothelial cells (LSECs) $(4,17,18)$. VEGFR-2 has been known to be expressed in endothelial cells and some vascular tumors, but many reports have also noted expression in carcinomas and lymphomas (19). However, the mechanisms of angiogenesis and their association with VEGFR-2 during regression of liver fibrosis and liver regeneration are not fully understood.

The present study focused on examining the critical role of VEGFR-2 in liver damage conditions induced by thioacetamide (TAA). To elucidate the role of VEGFR-2 based on three liver conditions, the mice were divided into three groups as follows: the control group, the TAA group, and the TAA-R group. The relative patterns VEGFR-2 expression in the different liver conditions were analyzed according to TAA administration and the results elucidated the role of VEGFR-2 in each liver state. We administrated Dulbecco's PBS or TAA to induce liver fibrosis and included a regeneration period.

\section{Materials and Methods}

Animals and experimental design. Nine-week-old male C57BL/6 mice weighing $25 \mathrm{~g}$ each were housed in individually ventilated cages that were maintained at $22^{\circ} \mathrm{C}-24^{\circ} \mathrm{C}$ and $40 \%-50 \%$ humidity under a 12-h light-dark cycle. Normal chow diet and water were provided ad libitum. The animals were acclimated in this environment for two weeks before the experiment. The 16 mice were divided into three groups. The control group mice were injected with saline intraperitoneally. The first experimental group was intraperitoneally injected with TAA (Sigma, St. Louis, MO, USA) for 17 weeks (TAA group), and the second experimental group was intraperitonealy injected with TAA for 17 weeks, followed by an additional regeneration period for 2 weeks (TAA-R group). The dosages for the intraperitoneal TAA route ranged from $50 \mathrm{mg} / \mathrm{kg}$ to a maximum dose of $300 \mathrm{mg} / \mathrm{kg}$. TAA was administered at a dose of $50 \mathrm{mg} / \mathrm{kg}$ twice per week for 10 weeks. After 10 weeks, TAA was given three times per week, with the dose increasing by $50 \mathrm{mg} / \mathrm{kg}$ a week until reaching $300 \mathrm{mg} / \mathrm{kg}$ for the last three weeks. For 17 weeks, saline was given to the control group whereas TAA was given the TAA and TAA-R groups. The control and TAA groups were euthanized two days after the last injection. After receiving of the last dose of TAA, the TAA-R group underwent twoweek regeneration period before being euthanized. All animal experiments were conducted in accordance with the National Institutes of Health (NIH) guidelines for the care and use of laboratory animals and were approved by the Kyungpook National
Table I. Histological scoring system for the assessment of liver fibrosis.

\begin{tabular}{ll}
\hline Grade & \multicolumn{1}{c}{ Histological description } \\
\hline 0 & $\begin{array}{l}\text { No fibrosis } \\
1\end{array}$ \\
2 & $\begin{array}{l}\text { Centrilobular perisinusoidal/pericellular fibrosis } \\
\text { Centrilobular perisinusoidal/pericellular fibrosis and portal/ } \\
\text { periportal fibrosis }\end{array}$ \\
3 & $\begin{array}{l}\text { Centrilobular perisinusoidal/pericellular fibrosis and portal/ } \\
\text { periportal fibrosis with focal or extensive bridging fibrosis }\end{array}$ \\
4 & Cirrhosis
\end{tabular}

University Institutional Animal Care and Use Committee (IACUC, approval number 2019-0024 and 2019-0070).

Histopathology. For the histological analysis of liver tissues, the liver tissue was fixed in $10 \%$ neutral buffered formalin, routinely processed, and embedded in paraffin wax. The paraffin blocks were cut to a thickness of $3 \mu \mathrm{m}$. The sections were deparaffinized with toluene for hematoxylin and eosin (H\&E) or Masson's trichrome (MT) staining. The tissue samples were rehydrated with graded ethanol solutions, washed in distilled water, and stained. A histological analysis of fibrosis was performed using a modified liver fibrosis grading system (grades $0 \sim 4$, Table I). The stained tissue slides were observed, and representative images were captured using a Leica microscope (Leica Microsystems, Heerbrugg, Switzerland).

Immunofluorescence. For the immunofluorescence analysis, the collected liver tissues were fixed in $4 \%$ paraformaldehyde at $4{ }^{\circ} \mathrm{C}$ for 2 days in the dark and were then washed with PBS three times for $5 \mathrm{~min}$, followed by overnight incubation in $30 \%$ sucrose until the tissue samples were no longer floating. The tissue samples that were sufficiently incubated in sucrose were embedded in OCT compound (Sakura FineTek, Torrance, CA, USA) and rapidly frozen on dry ice. The frozen OCT blocks were cryo-sectioned a thickness of $5 \mu \mathrm{m}$ by a cryostat. The sections were immunostained with primary antibodies of rabbit anti-VEGFR-2 (Cell Signaling, Danvers, MA, USA), and mouse anti-PCNA (Santa Cruz Biotechnology, Santa Cruz, CA, USA). The sections were subsequently incubated with the secondary antibody Alexa Fluor ${ }^{\circledR}$ 555 donkey anti-rabbit IgG (Abcam, Cambridge, MA, USA). ProLong ${ }^{\circledR}$ Gold Antifade Reagent with DAPI (Cell Signaling) was used for the nuclear staining. VEGFR-2 was observed under an Olympus BX53 fluorescence microscope (Olympus, Tokyo, Japan) at wavelength of $555 \mathrm{~nm}$. The immunostained tissues were observed under an Olympus BX53 fluorescence microscope using ToupView software (version $\times 86,3.7 .7817$, Hangzhou ToupTek Photonics Co., Zhejiang, PR China).

Immunoblot analysis. The frozen liver tissues were homogenized in activated VJ lysis buffer containing sodium fluoride $(\mathrm{NaF})$, sodium pyrophosphate $\left(\mathrm{Na}_{4} \mathrm{P}_{2} \mathrm{O}_{7}\right), 0.1 \mathrm{mM}$ sodium vanadate $\left(\mathrm{Na}_{3} \mathrm{VO}_{4}\right)$, a protease inhibitor cocktail tablet (Roche Diagnostics, Mannheim, Germany), and Pefabloc SC (Roche). The homogenized liver tissues were centrifuged at $6,000 \mathrm{rpm}$ and $4^{\circ} \mathrm{C}$ for $10 \mathrm{~min}$. The clear supernatant was centrifuged at $13,000 \mathrm{rpm}$ and $4^{\circ} \mathrm{C}$ for $20 \mathrm{~min}$. The lipids in the upper layer were discarded and the proteins in the lower 
layer were collected. The protein concentrations were measured using a Bio-Rad protein assay kit (Bio-Rad, Hercules, CA, USA). A $100 \mu \mathrm{g}$ aliquot of protein mixed with $1 \mathrm{X}$ electrophoresis sample buffer and lysis buffer was boiled for 10 min using a dry bath incubator. The boiled protein samples were loaded onto a $10 \%$ acrylamide gel for electrophoresis and then transferred to polyvinylidene difluoride (PVDF) membranes (Millipore Corp, Bedford, MA, USA). The transferred membranes were blocked with 5\% skim milk diluted in Tris-buffered saline containing $0.1 \%$ Tween20 (TBS/T) for $1 \mathrm{~h}$ and then incubated with the following antibodies: rabbit anti-VEGFR-2 (Cell Signaling), rabbit anti-VEGF (Santa Cruz Biotechnology), rabbit anti-SMP30 (COSMOBIO CO., LTD., Tokyo, Japan), and mouse anti-PCNA (Santa Cruz Biotechnology). $\beta$-actin (Sigma-Aldrich) was used as an internal standard. After incubation with the primary antibodies, the membranes were incubated with horseradish peroxidase (HRP) conjugated goat-anti-rabbit or goatanti-mouse antibodies (Calbiochem, San Diego, CA, USA) and diluted in 5\% skim milk for $1 \mathrm{~h}$ at room temperature. The antigenantibody complex was visualized using the ProNA ${ }^{\mathrm{TM}}$ ECL Ottimo (Translab, Seoul, Republic of Korea), and the images were recorded using Amersham ${ }^{\mathrm{TM}}$ Imager 680 (GE Healthcare Bjorkgatan, Uppsala, Sweden).

Serum biochemistry for the measuring ALT. Whole blood was collected from all mice and centrifuged at $3000 \mathrm{rpm}$ for $15 \mathrm{~min}$ at $4^{\circ} \mathrm{C}$. The supernatant was then collected. Serum triglyceride levels were measured using a spectrophotometer and an ALT activity assay kit (BioVision, Milpitas, CA, USA). Each mouse serum sample was mixed with master mix in a 96-well plate and incubated for $10 \mathrm{~min}$ at $37^{\circ} \mathrm{C}$, after which the absorbance was measured at $570 \mathrm{~nm}$.

cDNA synthesis and quantitative RT-PCR. Total liver RNA was extracted using TRIzol Reagent (Invitrogen, Carlsbad, CA, USA), and the RNA concentrations were measured by Nanodrop. The extracted RNA was used as a template for cDNA synthesis using the Advantage RT-for-PCR kit (Elpis Bio, Daejeon, Republic of Korea) with M-MLV Reverse Transcriptase, random hexamers and oligo dT. The synthesized cDNAs were mixed with SYBR Green PCR master mix (Elpis Bio) and 5 pmol of mouse-specific primers (Bioneer, Daejeon, Republic of Korea). The synthesized cDNA was mixed with SYBR green supermix (Bio-Rad Laboratories, Hercules, CA, USA), ultra-pure water and primers, followed by amplification using CFX maestro software.

In vitro experiments. Alpha mouse liver 12 (AML12) mouse hepatocyte cell lines were cultured at $37^{\circ} \mathrm{C}$ in Dulbecco's modified Eagle's medium and Ham's F-12 nutrient 1:1 mixture (DMEM/F12) (WELGENE Inc., Gyeongsan, Republic of Korea) supplemented with $10 \%$ heat -inactivated fetal bovine serum (FBS) (Invitrogen, Carlsbad, CA, USA), $1 \times$ insulin transferrin-selenium-pyruvate solution (WELGENE Inc.), $1 \times$ antibiotic-antimycotic solution (WELGENE Inc.), and dexamethasone $(100 \mathrm{nM})$ in a $37^{\circ} \mathrm{C}, 5 \% \mathrm{CO} 2$ incubator. Hepatoma G2 human hepatocellular carcinoma cell lines were cultured at $37^{\circ} \mathrm{C}$ in Dulbecco's modified Eagle's medium (WELGENE Inc.) with 10\% heat inactivated FBS (Invitrogen) and $1 \times$ antibiotic-antimycotic solution (WELGENE Inc.). The AML12 and $\mathrm{HepG} 2$ cells were seeded in 12 -well plates at a density of $5 \times 10^{4}$ cells per well. After $24 \mathrm{~h}$, the cells were treated with $100 \mathrm{mM}$ TAA (Sigma) diluted in DPBS (WELGENE Inc.). The cells were then fixed in $4 \%$ paraformaldehyde at room temperature for 20 min with shaking and washed three times in PBS for $5 \mathrm{~min}$. The fixed cells were blocked in $5 \%$ donkey serum for $1 \mathrm{~h}$ and then incubated with primary monoclonal rabbit anti-VEGFR-2 (Cell Signaling) diluted in $0.1 \%$ triton X. The cells were incubated with Alexa Fluor ${ }^{\circledR} 555$ donkey anti-rabbit IgG (Abcam) secondary antibodies. ProLong ${ }^{\circledR}$ Gold Antifade Reagent with DAPI (Cell Signaling) was used for nuclear staining. VEGFR-2 was observed using an Olympus BX53 fluorescence microscope (Olympus) at $555 \mathrm{~nm}$ using ToupView software (version $\times 86,3.7 .7817$, Hangzhou ToupTek Photonics Co.).

Isolation and culture of primary hepatocytes. Primary hepatocytes were isolated using a liver perfusion system. The liver was perfused with digestion buffer and then homogenized and filtered through a $70 \mu \mathrm{m}$ nylon mesh filter. The non-parenchymal cell fractions were discarded after centrifugation at $50 \times \mathrm{g}$ for $3 \mathrm{~min}$ at room temperature. The hepatocyte pellet was resuspended with $50 \% 1 \mathrm{X}$ Percoll. After centrifugation at $50 \times \mathrm{g}$ for $5 \mathrm{~min}$, the hepatocytes were washed twice with basal maintenance medium. The isolated hepatocytes were cultured in adherence medium for $6 \mathrm{~h}$. The media was replaced with basal maintenance media. The hepatocytes were incubated in a $37^{\circ} \mathrm{C}, 5 \% \mathrm{CO}_{2}$ incubator. Primary hepatocytes were seeded in 12-well plates at a density of $5 \times 10^{4}$ cells per well, and after $24 \mathrm{~h}$, the cells were treated with $25 \mathrm{mM}$ TAA (Sigma) diluted in DPBS (WELGENE Inc.). Thereafter, immunofluorescence was performed with primary rabbit anti-VEGFR-2 antibodies (Cell Signaling) in the same manner as in the AML12 and HepG2 cells.

Statistical analysis. The data are presented as the mean \pm standard error. A two-way analysis of variance or the Student's $t$-test was used to determine the statistical significance between multiple experimental groups using GraphPad InStat (GraphPad Software, Inc., San Diego, CA, USA). Statistical significance was set at $p<0.05$.

\section{Results}

Livers of the TAA group exhibited greater hepatocellular nodules formation on the liver surface compared with the other groups. TAA was intraperitoneally injected into the experimental groups for 17 weeks. Interestingly, the livers of the TAA mice exhibited significantly more hepatocellular nodules on the surface compared with the other groups (Figure 1A). As expected, the nodules were significantly decreased in the TAA-R group. After the gross and phenotype examination, we performed a microscopic observation to confirm the phenotypic differences mentioned above through histopathological analysis. All TAA-treated groups (the TAA and TAA-R groups), particularly the TAA group revealed centrilobular necrosis and inflammatory cell infiltration. The TAA group displayed much more severe hepatocyte necrosis and inflammatory cell infiltration around the central vein area than the TAA-R group. The livers of the control group showed intact normal liver morphology (Figure 1A). Compared to the TAA group, the TAA-R group displayed significantly alleviated in fibrosis, as confirmed by Masson's trichrome staining. The TAA-R group was characterized by a reduced extracellular matrix area during the regeneration period after the TAA treatment (Figure 1A). To quantify the progression of liver fibrosis in each group, a histopathological analysis was performed using modified fibrosis grading system (Table I). As expected, no fibrosis was observed in the control group. However, the livers of the TAA group were characterized by thick collagen bundles that of the central veins to central veins, 
A

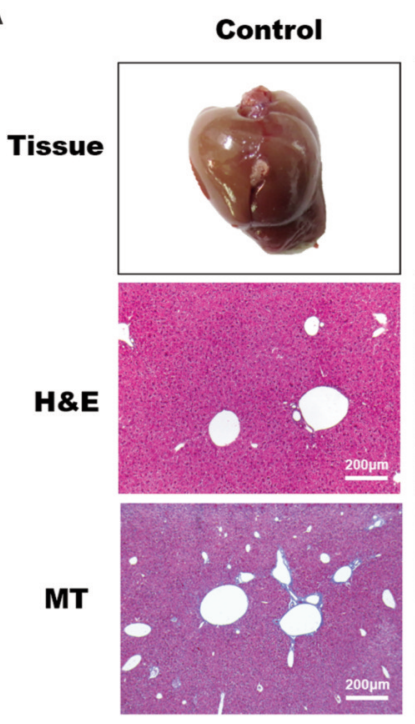

D
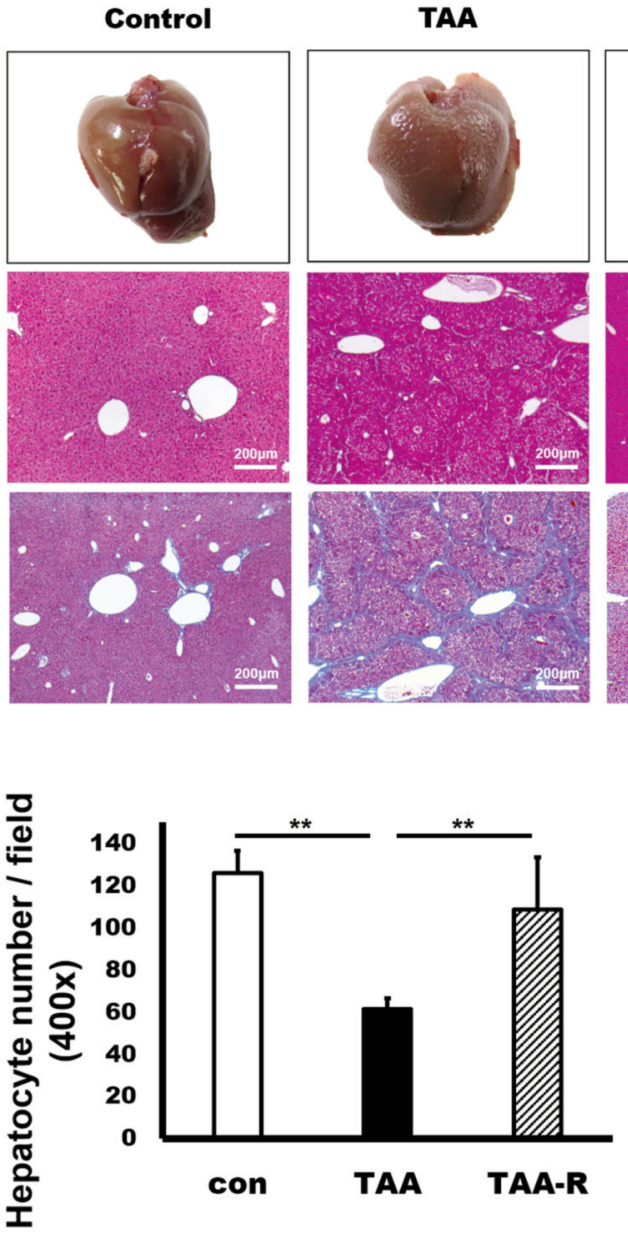

TAA-R

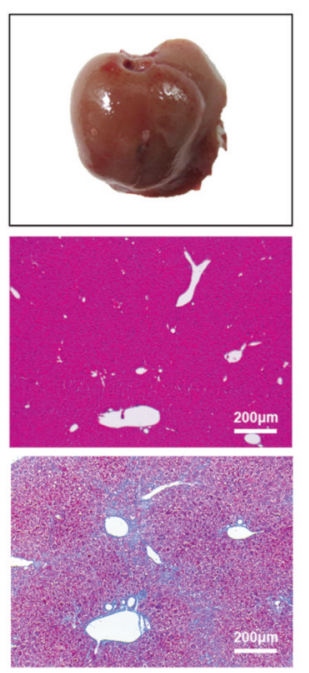

B

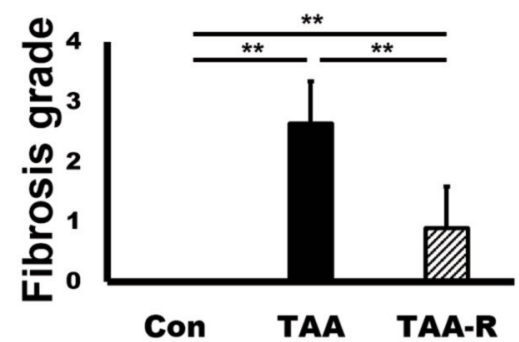

C

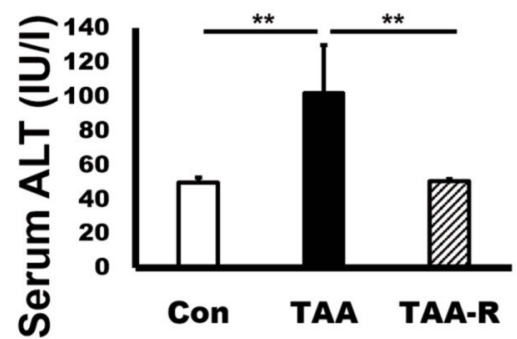

Figure 1. Histological characterization of livers after TAA administration. (A) Livers with intact periportal zones in the TAA group displayed hepatocellular surface nodules, side-spread centrilobular necrosis and inflammation. However, there were more hepatocellular nodules in the TAA group than in the TAA-R group. Furthermore, there was a prominent decrease in collagen fibers and the degree of inflammation in the livers of the TAA-R group. (B) The liver fibrosis grade was considerably increased in the TAA group. However, the TAA-R group showed reduced liver fibrosis grades compared to those of the TAA group. (C) The serum ALT level was dramatically decreased in the TAA-R group compared to the TAA group. (D), (E) The average size of hepatocytes was markedly increased in TAA-treated livers. Data are shown as the mean $\pm \mathrm{SD}(* p<0.05, * * p<0.01)$.

which extended to the fibrotic bridge. Some of the mice in the TAA group showed pre-cirrhotic conditions. The TAA-R group exhibited significantly alleviated liver fibrosis compared with the TAA group (Figure 1B). The serum ALT levels were then measured using an ALT activity assay kit. The serum ALT levels of the TAA group were significantly higher than those of the control group and twice as high as those of the TAA-R group. In fact, the ALT level of the TAA-R group was similar to that of the control (Figure 1C), which suggests that the liver in the TAA-R group underwent a regeneration period after severe injury and sufficiently recovered. The microscopic observation noted that the TAA group had larger but fewer hepatocytes. There were significantly fewer hepatocytes per field in the TAA group as a result of the TAA treatment. The TAA$\mathrm{R}$ group had more hepatocytes than the TAA group (Figure 1D). In agreement with the results mentioned above, the livers of the TAA group had significantly larger hepatocytes than those of the control group. The average size of the hepatocytes in the TAA-R group was increased compared to that of the control groups, but this difference was not significant. However, the hepatocytes in the TAA-R group were significantly reduced in size compared to the TAA group (Figure 1E). Taken together, these results indicate that, portion of the lost cells was replaced by regenerative hepatocytes causing the increment in the volume of organs or tissues even though the liver was damaged after TAA treatment.

Expression levels of fibrogenic and inflammatory $m R N A$ were dramatically increased in the TAA group, compared to the control and TAA-R groups. qRT-PCR was performed to quantify fibrosis of inflammatory-related gene expression. The mRNA expression of $\alpha-$ SMA, the marker of activated HSCs that plays an important role in 
A

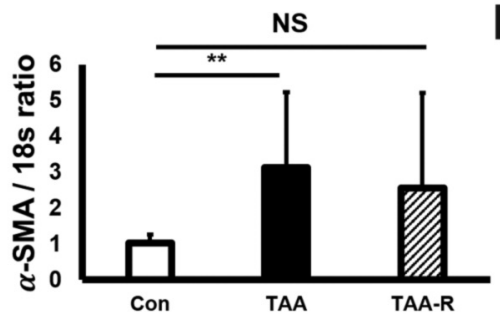

D

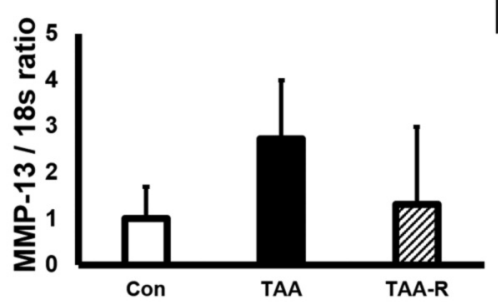

B

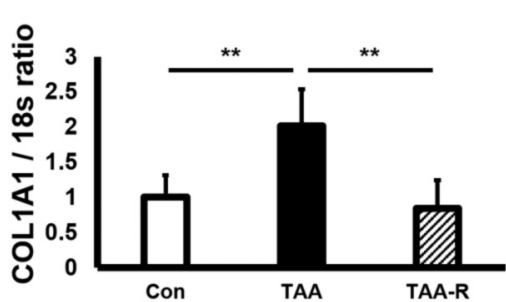

$E$

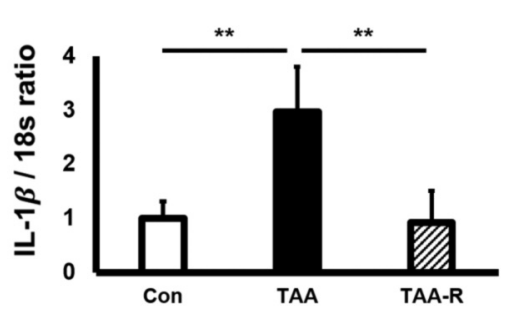

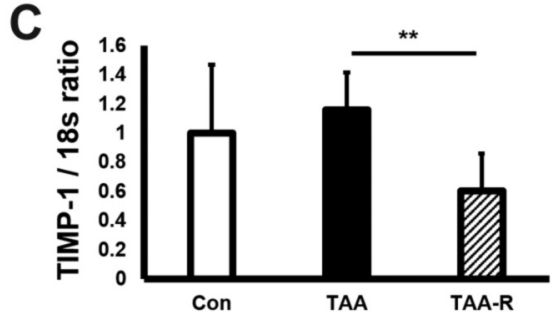

$\mathbf{F}$

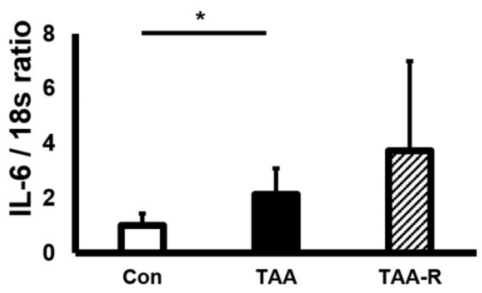

Figure 2. Effect of TAA on fibrogenic or inflammatory $m R N A$ expression. (A) Note the increasing trend of $\alpha-S M A$ expression in the TAA-treated group compared to the control group. However, there were no significant differences between the control and TAA-R groups. (B-F). Only the TAA group exhibited significantly increased expression of COL1A1, IL-1 $\beta$, TIMP-1 and MMP-13, respectively, as compared with the other groups. (D) The TAA-treated group showed increased IL-6 expression compared to the control group. The TAA-R group displayed the highest value among all groups, but this difference was not significant. Data are shown as the mean $\pm S D(* p<0.05, * * p<0.01)$.

the progression of liver fibrosis, was higher in the two groups that received TAA treatment compared to the control group (Figure 2A). However, there was no significant difference between the control group and the TAA-R group. COL1A1, a marker of a collagen type 1 produced by activated HSCs, was highly expressed in the TAA group (Figure 2B). Conversely, the control and TAA-R groups had similar mRNA expression levels. The expression of TIMP-1, a collagenase inhibitor, was significantly higher in the TAA group (Figure 2E). MMP-13, which codes for collagenase, showed the highest expression in the TAA group but there was no significant difference between the three groups (Figure 2F). Like COL1A1, TIMP-1 had a similar expression level in both the control and the TAA-R groups. IL-6, an inflammatory and proliferation marker, was significantly increased in the TAA group compared with the control group (Figure 2D). The TAA-R group had the highest average mRNA levels. UnlikeIL-6, IL-1 $\beta$, another inflammatory marker, was highly expressed in the TAA and control groups, but not in TAA-R. In particular, the TAA group displayed mRNA levels that were significantly increased compared with the TAA-R group (Figure 2C). This experiment demonstrates that the TAA group had marked liver fibrosis progression, and the TAA-R group displayed sufficient regeneration to a state similar to that of normal livers.

Dramatically increased VEGFR-2 expression was noted in the TAA$R$ group. VEGFR-2 was localized to the LSECs of the TAA group, whereas it was observed in the nucleus of hepatocytes in the TAA-R group during liver regeneration. Immunoblot analyses were performed to investigate the expression and quantities of VEGF and VEGFR-2. VEGFR-2 showed very weak expression in the control group and was rarely expressed in the TAA group. Conversely, VEGFR-2 was significantly expressed in the TAA-R group (Figure $3 A)$. The TAA-R group showed significantly increased levels of VEGFR-2 expression compared to the other groups (Figure 3A). Specially, VEGFR-2 expression levels in the TAA-R group were more than seven times higher than those in the control group (Figure 3B). These results indicate that VEGFR-2 expression can be altered according to different liver conditions, particularly during liver regeneration. To identify the types and the location of the cells that specifically express VEGFR-2, we performed an immunofluorescence assay using an anti-VEGFR-2 antibody. In the control group, VEGFR-2-positive cells were hepatocytes, but the expression level of VEGFR-2 was undetectably low. There was a huge difference in the expression patterns of VEGFR-2 between the two TAA-treated groups. In the TAA group, most VEGFR-2-positive cells were LSECs, whereas the VEGFR-2-positive cells were hepatocytes in the TAA-R group. Interestingly, VEGFR-2 expression in these two groups not only differed in the types of cells expressing VEGFR-2, but also in the intracellular location. There was a difference in the expression levels of VEGFR-2 between the control group and the TAA-R group, but the expression cells and the intracellular location were the same (Figure 3C). In these two groups, VEGFR-2 was specifically expressed in the nucleus of hepatocytes. VEGFR-2 expression was more clearly identified using a confocal microscope (Figure 3D). High levels of VEGFR-2 expression in the nucleus of hepatocytes, particularly in the TAA-R group, were very characteristic. Thus, depending on the different conditions of the liver, the cells that are rapidly proliferating may differ. VEGFR-2 expression appears to increase in those rapidly proliferating cells.

Nuclear expression of VEGFR-2 was significantly increased in TAAtreated AML12 and HepG2 cells. Based on the in vivo results, we assumed that VEGFR-2 expression in the nuclei promoted cell proliferation. Thus, we performed a cell culture experiment to revalidate this finding in vitro (Figure $4 \mathrm{~A}$ and $\mathrm{D}$ ). Consistent with the in vivo experiment, VEGFR-2 was rarely expressed in the control wells seeded with normal mouse hepatocytes (AML12 cells) (Figure 4B). However, in the TAA and TAA-R wells in which the cells were actively proliferating, the expression level of VEGFR-2 


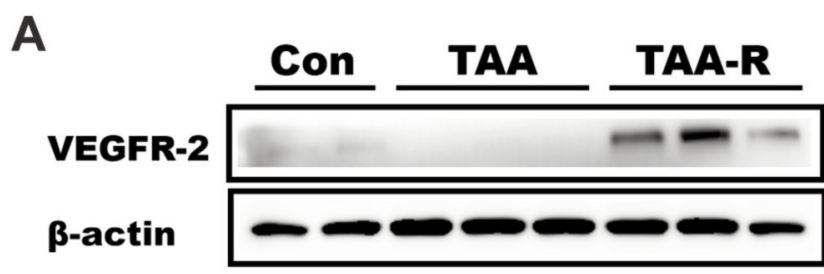

C
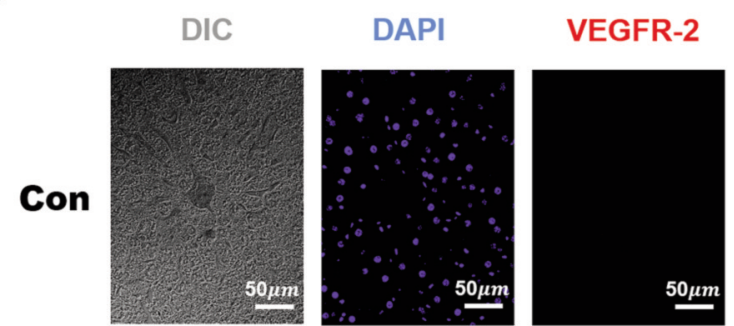

Merged
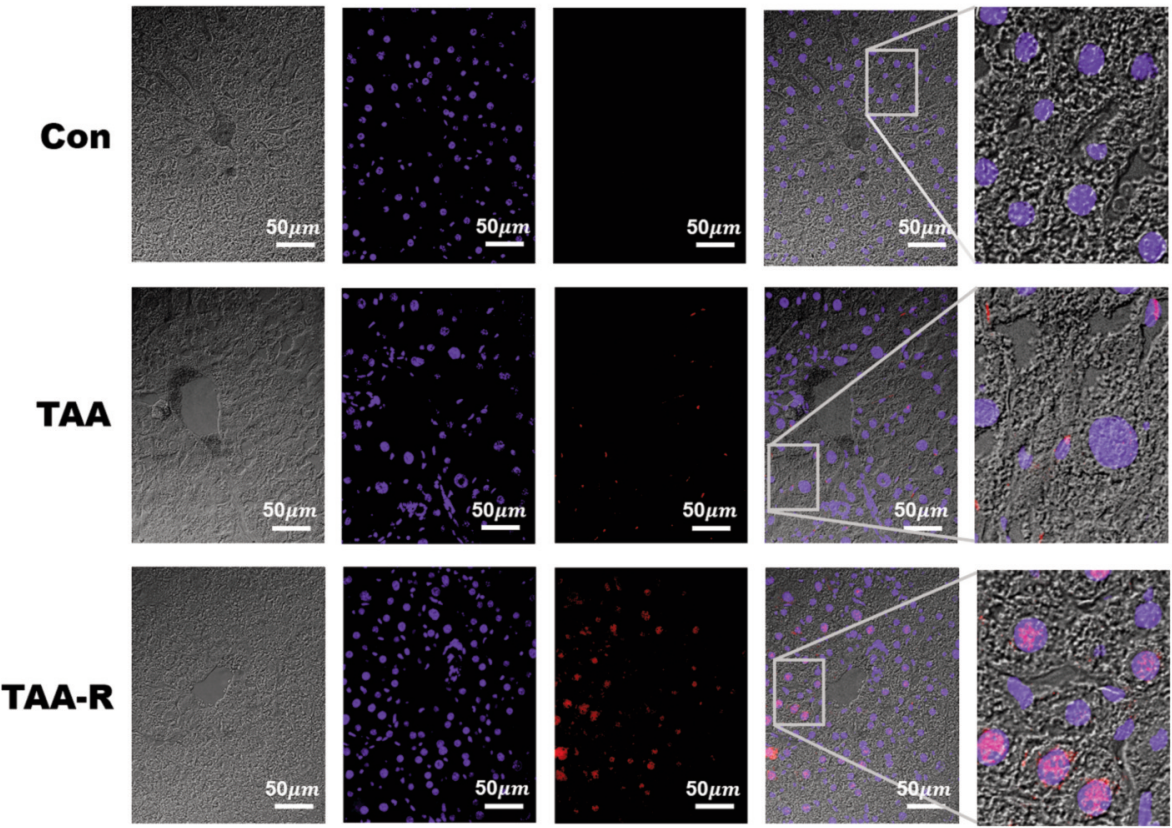
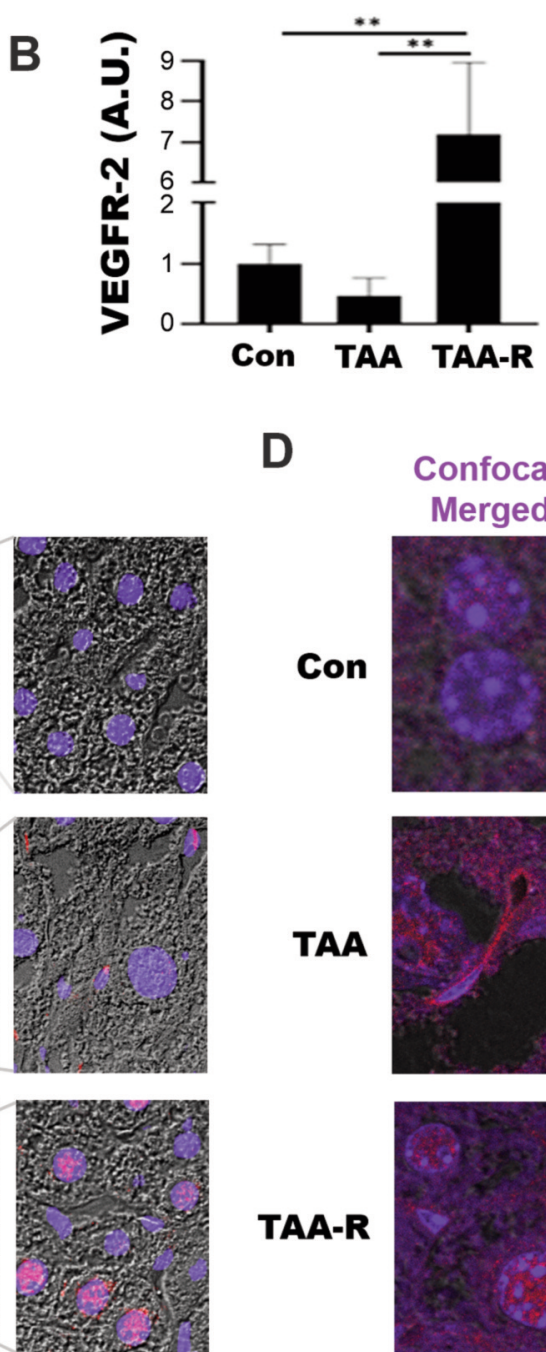

D

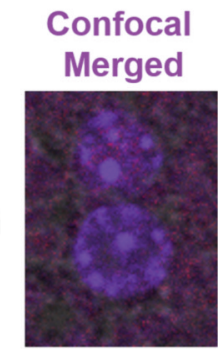

TAA

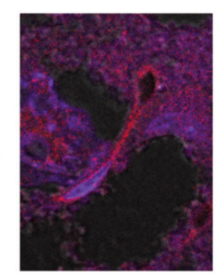

TAA-R

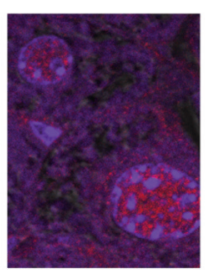

Figure 3. Immunoblot analysis of the expression level of VEGFR-2 and the location of VEGFR-2 expression in the TAA and TAA-R groups. (A) Immunoblot analysis for VEGFR-2 and $\beta$-actin. The TAA-R group displayed significantly increased VEGFR-2 expression compared to the other groups. (B) Relative immunoblot expression level of VEGFR-2. The expression of VEGFR-2 was extremely high in the TAA-R group compared to the other groups. The graph represents the band density relative to $\beta$-actin. (C) Representative immunofluorescence micrographs of VEGFR-2 (red). VEGFR-2 was mainly distributed in the cytoplasm of LSECs in the TAA group and in the nuclei of hepatocytes in the control and TAA-R groups. (D) Confocal microscopy was used to confirm VEGFR-2 expression. Scale bar=50 $\mu \mathrm{m}$. Blue=DAPI; Gray=DIC; Red=VEGFR-2. Data are shown as the mean $\pm S D(* p<0.05, * * p<0.01)$.

was increased in the nucleus of hepatocytes. The expression levels of VEGFR-2 in the nuclei of hepatocytes were much higher than in the TAA wells, particularly in the TAA-R wells (Figure 4B and C). Therefore, we confirmed that VEGFR-2 was expressed in the nuclei of rapidly proliferating AML12 cells, which are mouse liver cells. To confirm this finding in human cells, HepG2, a human hepatocellular carcinoma cell line, was used. VEGFR-2 was expressed in the cytoplasm of HepG2 cells in the control wells (Figure 4E). Conversely, in the two conditions of HepG2 cells treated with TAA, VEGFR-2 expression was observed in both the cytoplasm and the nuclei. In particular, VEGFR-2 showed significantly higher expression levels in TAA-R wells than in the other wells (Figure 4E and F). Thus, in the in vitro experiments,
VEGFR-2 expression was significantly increased in the nuclei of highly proliferative cells (Figure 4B and E).

Nuclear expression of VEGFR-2 was highly increased in the nuclei of regenerative hepatocytes. For further confirmation, we isolated primary hepatocytes from mouse livers by performing liver perfusion and digestion (Figure 5A). In general, VEGFR-2 was rarely expressed in the control wells consisting of normal hepatocytes. However, notable nuclear VEGFR-2 expression in hepatocytes was observed under TAA treatment conditions in the same manner as in the AML12 and HepG2 cells (Figure 5B, C). These results indicated that the expression of VEGFR-2 increases significantly in the nuclei of hepatocytes during the recovery process after damage. 
A

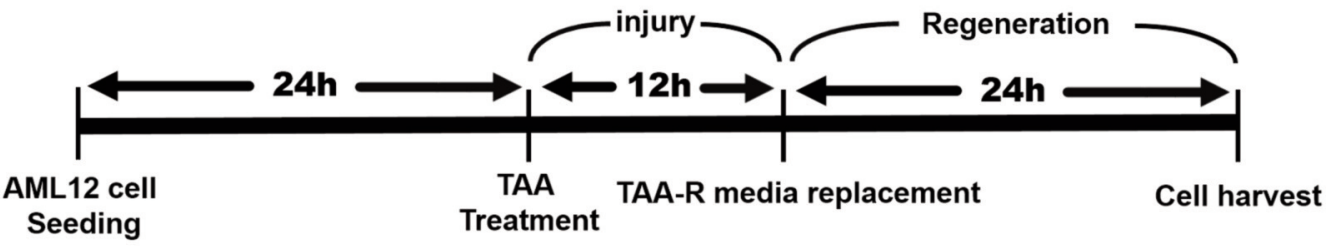

B

DAPI

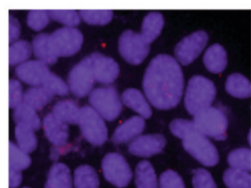

TAA

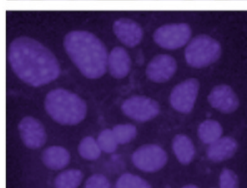

TAA-R

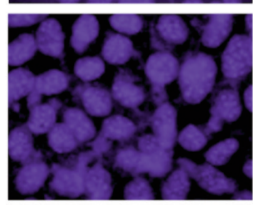

VEGFR-2
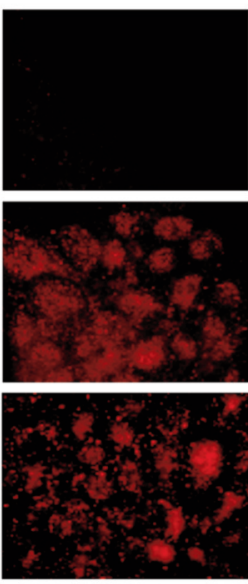

Merged

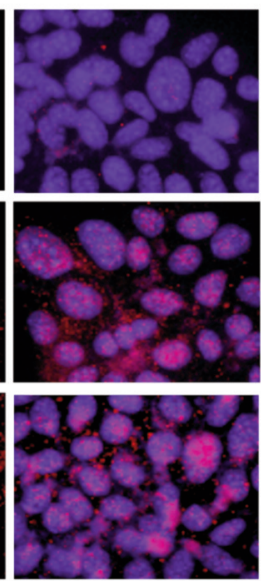

C

Confocal

Con

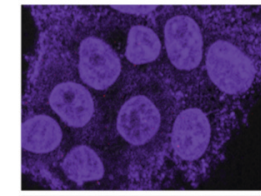

TAA

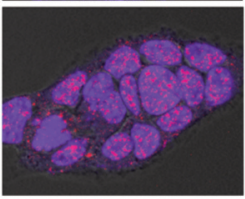

TAA-R

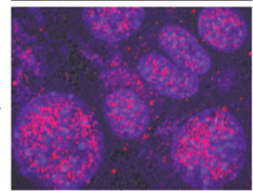

D

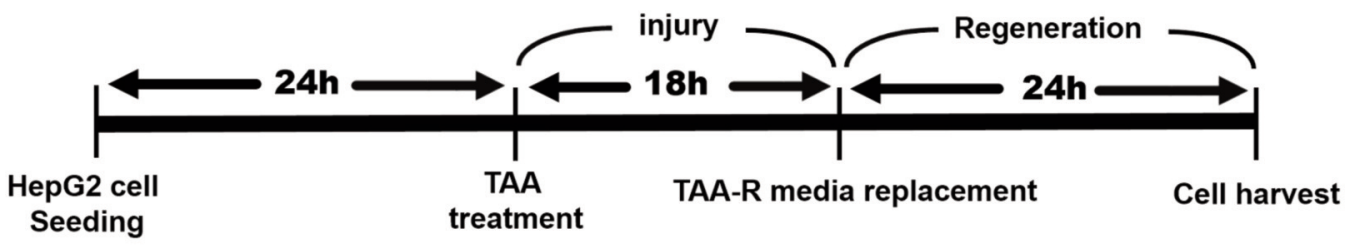

E

\section{DAPI}

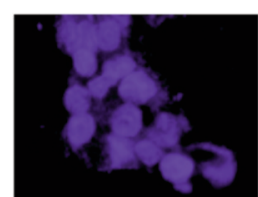

TAA

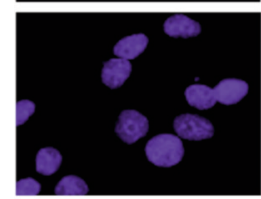

TAA-R

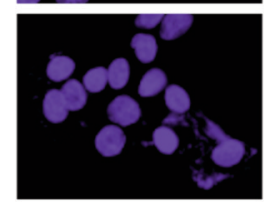

VEGFR-2
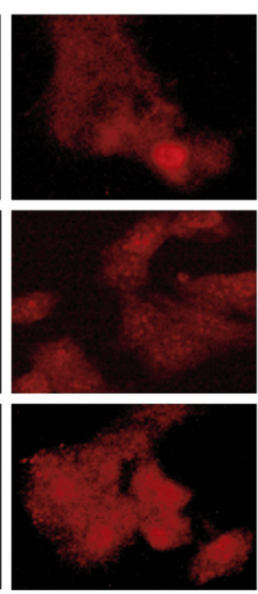

Merged
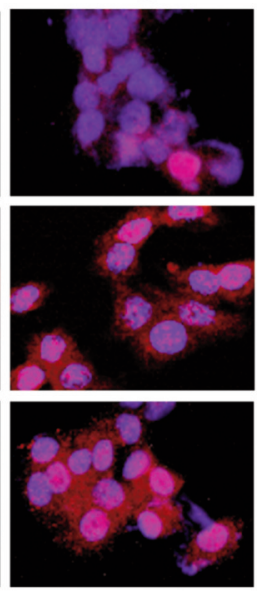

F

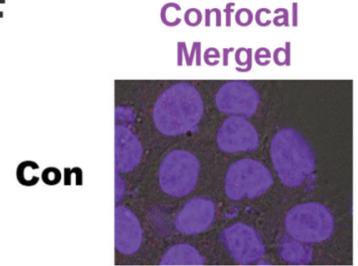

TAA

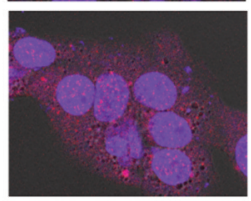

TAA-R

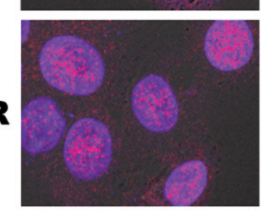

Figure 4. VEGFR-2 expression levels were highly elevated in TAA-treated AML12 and HepG2 cells. (A) Experimental design of the in vitro experiment using AML12 cells. (B) VEGFR-2 was mainly expressed in the nuclei of AML12 cells in the TAA and TAA-R groups in the rapidly proliferating cells. $(C),(F)$ VEGFR-2 expression of VEGFR-2 was confirmed by confocal microscopy. (D) At 12 h after TAA treatment, the population of HepG2 cells was reduced by 50\%. (E) VEGFR-2 was expressed only in the cytoplasm of HepG2 cells in the control group. VEGFR-2 was mainly distributed in the nuclei of HepG2 cells in the TAA and TAA-R groups in the rapidly proliferating cells. Blue=DAPI; Red=VEGFR-2. 


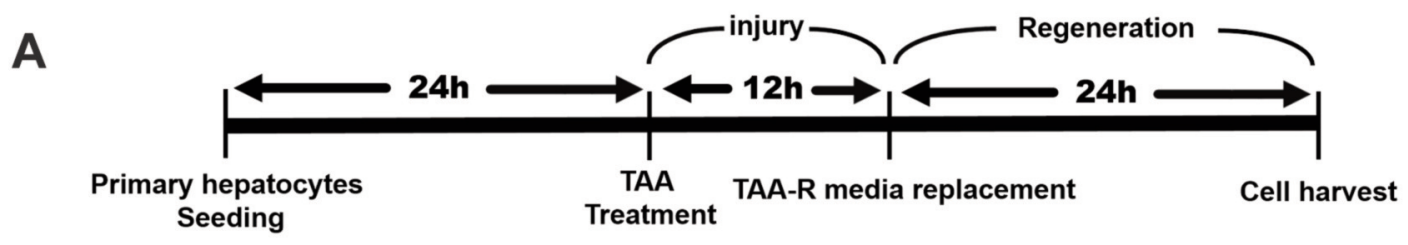

B

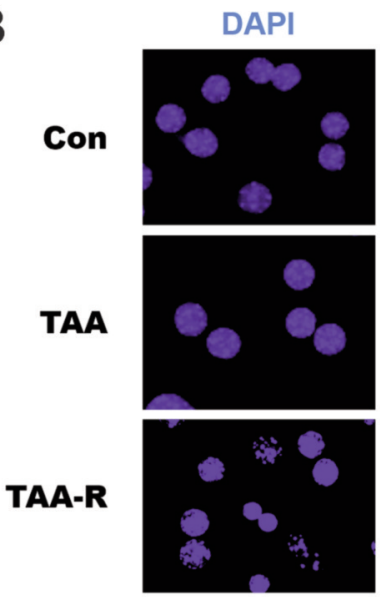

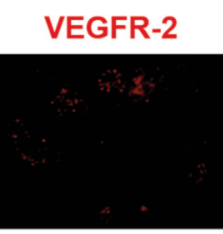

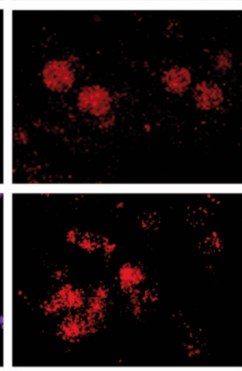

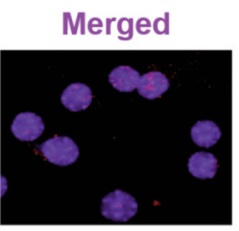

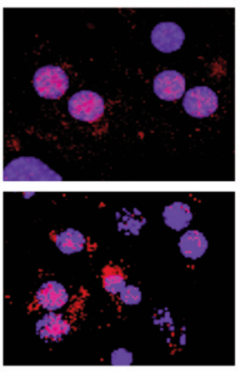

C

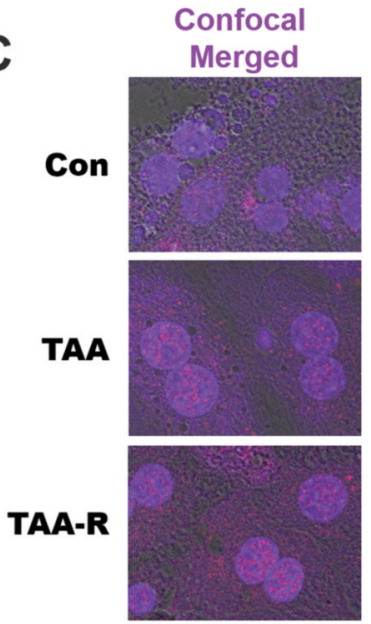

Figure 5. Nuclear expression of VEGFR-2 was dramatically increased in the TAA-treated primary hepatocytes. (A) VEGFR-2 expression was verified by primary hepatocytes. (B) VEGFR-2 was mainly distributed in the nuclei of primary hepatocytes in the TAA and TAA-R groups. (C) Confocal microscopy merged images showed that VEGFR-2 was expressed in the nuclei of primary hepatocytes in TAA and TAA-R groups. Blue=DAPI; Red=VEGFR-2; right panel=confocal.

\section{Discussion}

The present study demonstrated, for the first time, that nuclear expression of VEGFR-2 in hepatocytes is involved in liver regeneration. VEGFR-2 expression in the nuclei was also observed in rapidly proliferating cells and in LSECs. We found that VEGFR-2 plays an important role in liver regeneration and cell proliferation.

Previous studies have shown that VEGFR-2 is mainly expressed in the cytoplasmic membrane of endothelial cells (13). When hepatocytes in the liver are damaged, VEGFs are released and bind to VEGFR of endothelial cells to induce the ERK signalling pathway (20), resulting in the migration and proliferation of endothelial cells (21-23). VEGFRs are generally known to be expressed in endothelial cells, but recent studies have reported their expression in carcinomas and lymphomas (19). The presence of VEGFR-2 in cancer cells has been identified in breast $(24,25)$, lung (26), and gastric tumors (27). Although the cytoplasmic expression of VEGFR-2 in cancer cells has been considered a therapeutic target, this expression in the nuclei has not yet been considered (28). Furthermore, the role of VEGFR-2 expression in the nucleus of cells is not yet fully understood.

The VEGF/VEGFR-2 pathway is essential for effective liver regeneration. When hepatic VEGF levels increase from chronic injury or partial hepatectomy, bone marrow-derived sinusoidal endothelial progenitor cells are recruited into the liver (29). Additionally, LSECs promote hepatocellular growth factor expression through interaction of VEGF and VEGFR2 (30). In the present study, in vivo experiments showed that VEGFR-2 was rarely expressed in the control group, which was dominated by normal hepatocytes $(15,31)$. Conversely, VEGFR-2 showed a distinctive characteristic pattern in the livers of the TAA-treated groups (TAA and TAA-R). VEGFR2 expression was significantly increased in the TAA-R group (Figure 3). These expression levels showed different characteristics depending on the cell types and intracellular location for VEGFR-2 expression. VEGFR-2 was expressed mostly in LSECs in the TAA group; however, it was expressed in the nuclei of hepatocytes in the TAA-R group.

TAA is metabolized to thioacetamide sulfoxide (TASO) by CYP2E1, which is involved in the hypertrophy of nucleoli, increased intracellular $\mathrm{Ca}^{+2}$ concentrations, and cellular permeability $(32,33)$. It is then metabolized to thioacetamide sulfodioxide (TASO2). This causes nitric oxide synthesis, centrilobular necrosis, and protein denaturation $(33,34)$. Free radicals are also formed during the metabolism of TAA (35), inducing oxidative stress and cell necrosis (36). In the present study, the TAA group was characterized by fibrosis with centrilobular necrosis and inflammatory cell infiltration 
(Figure 1A). Moreover, fibrotic liver is accompanied by hypoxia due to ROS damage to hepatocytes and LSECs (37). In this condition, many new blood vessels are formed to supply more oxygen and nutrients to the liver cells (38), which indicates that hypoxia is the main inducer of neovascularization (39). Angiogenesis has a decisive effect on tissue growth and regeneration (40). Therefore, in the TAA group, hepatic endothelial cells seemed to proliferate to generate neovascularization to repair liver damage induced by TAA. In the TAA group, VEGFR-2 was observed in the most actively proliferating LSEC for angiogenesis. Conversely, as the liver recovered in the TAA-R group, hepatocytes and liver parenchymal cells, actively proliferated (Figures 4 and 5). Under microscopic observation, TAA-R group had more hepatocytes than the TAA group. The liver has a notable ability to regenerate after injury and resize to match its host. Cellular proliferation begins in the periportal region and proceeds to the center of the lobules (41). In the TAA-R group, proliferation of hepatocytes actively occurs in order to recover after liver injury. The TAA-R group showed VEGFR-2 expression in the nuclei of hepatocytes. Therefore, VEGFR-2 was highly expressed in cells, and its expression locations varied depending on the liver conditions.

VEGFR-2 is not generally expressed in normal hepatocytes (15). When tested in AML12 mouse hepatocytes, the normal AML12 mouse hepatocytes did not express VEGFR-2. However, in the TAA-treated wells, VEGFR-2 expression was significantly increased (Figure 4A). In both the TAA and TAA-R wells, VEGFR-2 was expressed in the nuclei of hepatocytes. Surprisingly, VEGFR-2 expression was higher in the TAA-R group, which had more active cell proliferation. Therefore, VEGFR-2 expression in the nucleus resulted in the active cell proliferation necessary for the recovery of liver damage induced by TAA rather than being an effect of TAA alone. The same pattern appeared when the experiments were conducted with HepG2 cells (Figure 4B).

Recent studies have reported that VEGFR-2 is expressed in carcinoma cells (19). VEGFR-2 expression in HCC has been identified in the cytoplasm of hepatocytes (42). We demonstrated that the nuclear internalization of VEGFR-2 in the liver is necessary for post-damage regeneration. Furthermore, the nuclear positioning of VEGFR-2 in proliferative tumor cells may be involved in nuclear molecular mechanisms in which proteins contribute to tumor progression $(43,44)$. These studies suggest that VEGFR-2 is associated with signaling activity that contributes to amplification of cell proliferation and angiogenesis in liver injury and regeneration processes. This observation in liver regeneration is very important because it is likely to be helpful in treatment of a variety of serious liver diseases. The underlying mechanism by which VEGFR2 is transferred to the nucleus is not yet fully understood. However, recent studies have suggested that VEGFR2 can be internalized preferentially through the caveolar pathway and transported to caveosomes $(45,46)$ around the nucleus.

In summary, VEGFR-2 expression in the nucleus is associated with cell proliferation, which may promote the regeneration of damaged tissue during chronic liver disease. These results provide a strong possibility for the use of VEGFR-2 as a novel therapeutic target for the treatment of chronic liver disease. Further studies are necessary to investigate the underlying mechanism of nuclear VEGFR-2 expression in hepatocytes.

\section{Conflicts of Interest}

The Authors declare that they have no competing interests.

\section{Authors' Contributions}

ARL carried out the experiments, performed data collection, data analysis and wrote the article. SMB and SWL reviewed pathological data and analysed the data. TUK was involved in data collection. JEH, SB, SJP, THK and KSJ reviewed and revised the manuscript. SKC and JKP designed the project, supervised progress, performed data analysis, reviewed and revised the manuscript.

\section{Acknowledgements}

This work was partly supported by the National Research Foundation of Korea (NRF) grants funded by the Korea government, the Ministry of Education (grant number 2019R1I1A2A01060031).

\section{References}

1 Bataller R, DAVID A and Brenner D: Liver fibrosis. J Clin Invest 115(2): 209-218, 2005. PMID: 15690074. DOI: 10.1172/JCI24282

2 Park JK, Utsumi T, Seo YE, Deng Y, Satoh A, Saltzman WM and Iwakiri Y: Cellular distribution of injected PLGAnanoparticles in the liver. Nanomedicine 12(5): 1365-1374, 2016. PMID: 26961463. DOI: 10.1016/j.nano.2016.01.013

3 Bartneck M, Warzecha KT and Tacke F: Therapeutic targeting of liver inflammation and fibrosis by nanomedicine. Hepatobiliary Surg Nutr 3(6): 364-376, 2014. PMID: 25568860. DOI: $10.3978 /$ j.issn.2304-3881.2014.11.02

4 Bussolino F, Mantovani A and Persico G: Molecular mechanisms of blood vessel formation. Trends Biochem Sci 22(7): 251-6, 1997. PMID: 9255066 DOI: 10.1016/s0968-0004(97)01074-8

5 Thabut D and Shah V: Intrahepatic angiogenesis and sinusoidal remodeling in chronic liver disease: new targets for the treatment of portal hypertension? J Hepatol 53(5): 976-980, 2010. PMID: 20800926 DOI: $10.1016 /$ j.jhep.2010.07.004

6 Elpek and Gülsüm Ö: Angiogenesis and liver fibrosis. World journal of hepatology. World J Hepatol 7(3):377-391, 2015. PMID: 25848465. DOI: 10.4254/wjh.v7.i3.377

7 Lehman SE, Morris AS, Mueller PS, Salem AK, Grassian VH and Larsen SC: Silica nanoparticle-generated ROS as a predictor of cellular toxicity: Mechanistic insights and safety by design. Environ Sci Nano 3(1): 56-66, 2016. PMID: 26998307. DOI: 10.1039/C5EN00179 
8 Gille J, Khalik M, König V and Kaufmann R: Hepatocyte growth factor/scatter factor (HGF/SF) induces vascular permeability factor (VPF/VEGF) expression by cultured keratinocytes. J Invest Dermatol. 111(6): 1160-1165, 1998. PMID: 9856833. DOI: 10.1046/j.1523-1747.1998.00418.x

$9 \mathrm{Ni}$ D, Jiang D, Ehlerding EB, Huang $\mathrm{P}$ and Cai W: Radiolabeling silica-based nanoparticles via coordination chemistry: Basic principles, strategies, and applications. Acc Chem Res 51(3): 778-788, 2018. PMID: 29489335. DOI: 10.1021/acs.accounts.7b00635

10 Olszewska-Pazdrak B, Hein TW, Olszewska P and Carney DH: Chronic hypoxia attenuates VEGF signaling and angiogenic responses by downregulation of KDR in human endothelial cells. Am J Physiol Cell Physiol 296(5): C1162-C1170, 2009. PMID: 19244479. DOI: 10.1152/ajpcell.00533.2008

11 Bayne CE, Williams SB, Cooperberg MR, Gleave ME, Graefen M, Montorsi F, Novara G, Smaldone MC, Sooriakumaran P, Wiklund PN and Chapin BF: Treatment of the primary tumor in metastatic prostate cancer: Current concepts and future perspectives. Eur Urol 69(5): 775-787, 2016. PMID: 26003223. DOI: $10.1016 /$ j.eururo.2015.04.036

12 Ahmad K, Rabbani G, Baig MH, Lim JH, Khan ME, Lee EJ, Ashraf GM and Choi I: Nanoparticle-based drugs: A potential armamentarium of effective anti-cancer therapies. Curr Drug Metab 19(10): 839-846, 2018. PMID: 28831911. DOI: $10.2174 / 1389200218666170823115647$

13 Cébe-Suarez S, Zehnder-Fjällman A and Ballmer-Hofer K: The role of VEGF receptors in angiogenesis; complex partnerships. Cell Mol Life Sci 63(5): 601-615, 2006. PMID: 16465447. DOI: $10.1007 / \mathrm{s} 00018-005-5426-3$

14 Grandhi MS, Kim AK, Ronnekleiv-Kelly SM, Kamel IR, Ghasebeh MA and Pawlik TM: Hepatocellular carcinoma: From diagnosis to treatment. Surg Oncol 25(2): 74-85, 2016. PMID: 27312032. DOI: 10.1016/j.suronc.2016.03.002

15 Mu X, Español-Suñer R, Mederacke I, Affò S, Manco R, Sempoux C, Lemaigre FP, Adili A, Yuan D, Weber A, Unger $\mathrm{K}$, Heikenwälder $\mathrm{M}$, Leclercq IA and Schwabe RF: Hepatocellular carcinoma originates from hepatocytes and not from the progenitor/biliary compartment. J Clin Invest 125(10): 3891-3903, 2015. PMID: 26348897. DOI: 10.1172/JCI77995

16 Jakeman LB, Winer J, Bennett GL, Altar CA and Ferrara N: Binding sites for vascular endothelial growth factor are localized on endothelial cells in adult rat tissues. J Clin Invest 89(1): 244253, 1992. PMID: 1729274. DOI: 10.1172/JCI115568

17 Miettinen M, Rikala MS, Rys J, Lasota J and Wang ZF: Vascular endothelial growth factor receptor 2 (VEGFR2) as a marker for malignant vascular tumors and mesothelioma? immunohistochemical study of 262 vascular endothelial and 1640 NONVASCULAR TUMORS. Am J Surg Pathol 36(4) :629-639, 2012. PMID: 22314185. DOI: 10.1097/PAS.0b013e318243555b

18 Ferrara N, Gerber HP and LeCouter J: The biology of VEGF and its receptors. Nat Med 9(6):669-676, 2006. PMID: 12778165. DOI: $10.1038 / \mathrm{nm} 0603-669$

19 Eichmann A and Simons M: VEGF signaling inside vascular endothelial cells and beyond. Curr Opin Cell Biol 24(2): 18893, 2012. PMID: 22366328. DOI: 10.1016/j.ceb.2012.02.002

20 Cheng SH, Li FC, Souris JS, Yang CS, Tseng FG, Lee HS, Chen CT, Dong CY and Lo LW: Visualizing dynamics of sub-hepatic distribution of nanoparticles using intravital multiphoton fluorescence microscopy. ACS Nano 6(5): 4122-4131, 2012. PMID: 22486639. DOI: 10.1021/nn300558p

21 Poisson J, Lemoinne S, Boulanger C, Durand F, Moreau R, Valla D and Rautou PE: Liver sinusoidal endothelial cells: Physiology and role in liver diseases. J Hepatol 66(1): 212-227, 2017. PMID: 27423426. DOI: 10.1016/j.jhep.2016.07.009

22 Braet F and Wisse E: Structural and functional aspects of liver sinusoidal endothelial cell fenestrae: A review. Comp Hepatol 1(1): 1, 2002. PMID: 12437787. DOI: 10.1186/1476-5926-1-1

23 DeLeve LD: Liver sinusoidal endothelial cells in hepatic fibrosis. Hepatology 61(5): 1740-1746, 2015. PMID: 25131509. DOI: $10.1002 /$ hep. 27376

24 Schroeder A, Levins CG, Cortez C, Langer R and Anderson DG: Lipid-based nanotherapeutics for siRNA delivery. J Intern Med 267(1): 9-21, 2010. PMID: 20059641. DOI: 10.1111/j.13652796.2009.02189.x

25 Poelstra K, Prakash J and Beljaars L: Drug targeting to the diseased liver. J Control Release 161(2): 188-197, 2012. PMID: 22370583. DOI: 10.1016/j.jconrel.2012.02.011

26 Khushbu R, Sawan Kumar J and Michael J: Biology of vascular endothelial growth factor $\mathrm{C}$ in the morphogenesis of lymphatic vessels. Front Bioeng Biotechnol 6:7, 2018. PMID: 29484295. DOI: 10.3389/fbioe.2018.00007

27 Srinivasan R, Zabuawala, Huang H, Zhang J, Gulati P, Fernandez S, Karlo JC, Landreth GE, Leone G and Ostrowski MC: Erk1 and Erk 2 regulate endothelial cell proliferation and migration during mouse embryonic angiogenesis. PLoS One 4(12): e8283, 2009. PMID: 20011539. DOI: 10.1371/journal.pone.0008283

28 Almalki SG and Agrawal DK: ERK signaling is required for VEGFA/VEGFR2-induced differentiation of porcine adiposederived mesenchymal stem cells into endothelial cells. Stem Cell Res Ther 8(1): 113, 2017. PMID: 28499402. DOI: 10.1186/s13287017-0568-4

29 Beljaars L, Molema G, Weert B, Bonnema H, Olinga P, Groothuis GM, Meijer DK and Poelstra K: Albumin modified with mannose 6-phosphate: A potential carrier for selective delivery of antifibrotic drugs to rat and human hepatic stellate cells. Hepatology 29(5): 1486-1493, 1999. PMID: 10216133. DOI: $10.1002 /$ hep. 510290526

30 Ding BS, Nolan DJ, Butler JM, James D, Babazadeh AO, Rosenwaks Z, Mittal V, Kobayashi H, Shido K, Lyden D, Sato TN, Rabbany SY and Rafii S: Inductive angiocrine signals from sinusoidal endothelium are required for liver regeneration. Nature 468(7321): 310-315, 2010. PMID: 21068842. DOI: 10.1038/nature09493

31 Wang L, Wang X, Xie G, Wang L, Hill CK and DeLeve LD: Liver sinusoidal endothelial cell progenitor cells promote liver regeneration in rats. J Clin Invest 122(4): 1567-1573, 2012. PMID: 22406533. DOI: 10.1172/JCI58789

32 Sarin H: Physiologic upper limits of pore size of different blood capillary types and another perspective on the dual pore theory of microvascular permeability. J Angiogenes Res 2: 14, 2010. PMID: 20701757. DOI: 10.1186/2040-2384-2-14

33 Riquelme E, Suraokar M, Behrens C, Lin HY, Girard L and Nilsson MB: VEGF/ VEGFR-2 upregulates EZH2 expression in lung adenocarcinoma cells and EZH2 depletion enhances the response to platinum-based and VEGFR-2-targeted therapy. Clin Cancer Res 20(14): 3849-3861, 2014. PMID: 24850841. DOI: 10.1158/1078-0432.CCR-13-1916 
34 Rensen PC, Sliedregt LA, Ferns M, Kieviet E, van Rossenberg $\mathrm{SM}$, van Leeuwen $\mathrm{SH}$, van Berkel TJ and Biessen EA: Determination of the upper size limit for uptake and processing of ligands by the asialoglycoprotein receptor on hepatocytes in vitro and in vivo. J Biol Chem 276(40): 37577-37584, 2001. PMID: 11479285. DOI: 10.1074/jbc.M101786200

35 Gómez-Suárez C, Bruinsma GM, Rakhorst G, van der Mei HC and Busscher HJ: Hydrophobicity of peritoneal tissues in the rat. J Colloid Interface Sci 253(2): 470-471, 2002. PMID: 16290879. DOI: $10.1006 /$ jcis.2002.8569

36 Sadauskas E, Wallin H, Stoltenberg M, Vogel U, Doering P, Larsen A and Danscher G: Kupffer cells are central in the removal of nanoparticles from the organism. Part Fibre Toxicol 4: 10, 2007. PMID: 17949501. DOI: 10.1186/1743-8977-4-10

37 Beljaars L, Molema G, Schuppan D, Geerts A, De Bleser PJ, Weert B, Meijer DK and Poelstra K: Successful targeting to rat hepatic stellate cells using albumin modified with cyclic peptides that recognize the collagen type VI receptor. J Biol Chem 275(17): 12743-12751, 2000. PMID: 10777570. DOI: 10.1074/ jbc. 275.17 .12743

38 Beljaars L, Weert B, Geerts A, Meijer DK and Poelstra K: The preferential homing of a platelet derived growth factor receptorrecognizing macromolecule to fibroblast-like cells in fibrotic tissue. Biochem Pharmacol 66(7): 1307-1317, 2003. PMID: 14505810. DOI: 10.1016/s0006-2952(03)00445-3

39 Oh H, Takagi H, Suzuma K, Otani A, Matsumura M and Honda Y: Hypoxia and vascular endothelial growth factor selectively up regulate angiopoietin-2 in bovine microvascular endothelial cells. J Biol Chem 274(22): 15732-15739, 1999. PMID: 10336473 DOI: $10.1074 /$ jbc.274.22.15732

40 Kai Wang, Xuejiao Chen, Yiwa Pan, Yun Cui, Xin Zhou, Deling Kong and Qiang Zhao: Enhanced vascularization in hybrid PCL/gelatin fibrous scaffolds with sustained release of VEGF. Biomed Res Int 2015: 865076, 2015. PMID: 25883978. DOI: $10.1155 / 2015 / 865076$
41 Kholodenko IV and Yarygin KN: Cellular mechanisms of liver regeneration and cell-based therapies of liver diseases. Biomed Res Int 2017: 8910821, 2017. PMID: 28210629. DOI: 10.1155/ 2017/8910821

42 Lee JA, Kim MK, Paek HJ, Kim YR, Kim MK, Lee JK, Jeong $\mathrm{J}$ and Choi SJ: Tissue distribution and excretion kinetics of orally administered silica nanoparticles in rats. Int $\mathrm{J}$ Nanomedicine 9(Suppl 2): 251-260, 2014. PMID: 25565843. DOI: $10.2147 /$ IJN.S57939

43 Blazquez C, Cook N, Micklem K, Harris AL, Gatter KC and Pezzella F: Phosphorylated KDR can be located in the nucleus of neoplastic cells. Cell Res 16(1): 93-98, 2006. PMID: 16467880. DOI: 10.1038/sj.cr.7310012

44 Fox SB, Turley H, Cheale M, Blázquez C, Roberts H, James N, Cook N, Harris A and Gatter K: Phosphorylated KDR is expressed in the neoplastic and stromal elements of human renal tumours and shuttles from cell membrane to nucleus. J Pathol 202(3): 313-320, 2004. PMID: 14991896. DOI: 10.1002/path.1520

45 Kapeller R, Chakrabarti R, Cantley L, Fay F and Corvera S: Internalization of activated platelet-derived growth factor receptor-phosphatidylinositol-3' kinase complexes: Potential interactions with the microtubule cytoskeleton. Mol Cell Biol 13(10): 6052-6063, 1993. PMID: 8413207. DOI: 10.1128/ mcb.13.10.6052

46 Labrecque L, Royal I, Surprenant DS, Patterson C, Gingras D and Béliveau R: Regulation of vascular endothelial growth factor receptor- 2 activity by caveolin- 1 and plasma membrane cholesterol. Mol Biol Cell 14(1): 334-347, 2003. PMID: 12529448. DOI: 10.1091/mbc.e02-07-0379

Received February 16, 2021

Revised March 6, 2021

Accepted March 12, 2021 